\title{
The mystical interlinks: Mechanics, religion or optics?
}

\author{
Hong-Bo Sun ${ }^{*}$ \\ State Key Laboratory on Integrated Optoelectronics, College of Electronic Science and Engineering, Jilin University, \\ Changchun 130012, China
}

Received November 6, 2015; accepted November 18, 2015

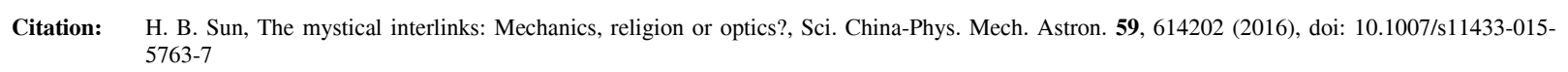

The beauty of this world relies on a fact that there is always this or that mystical interlink among different fields, one concept in a specific realm shines out with great brilliancy in another totally different territory. Catenary is the curve that a free-hanging chain assumes under its own weight, which is thought to be a "true mathematical and mechanical form" in architecture. The catenary shape could also be found in the silk of a spider's web, but no significant phenomena have ever been observed in optics.

$\mathrm{Pu}$ et al. [1] recently demonstrated that nanostructured catenaries could be used to construct ultrathin and lightweight optical devices, which are the key of the next-generation integrated photonic systems. This new finding is an excellent demonstration of how natural phenomena could inspire the development of modern optics. Interestingly, the operating bandwidth of the new optical devices could cover the entire electromagnetic spectrum ranging from microwave, terahertz, infrared, to the visible regime. Similar to the "catenary of equal strength", the phase gradient of the optical catenary is equal everywhere, which is a direct outcome of its special geometric shape. Based on the metasurface-assisted law of reflection and refraction, many novel optical elements, such as flat lenses, axicons, and prisms, could be realized with an excellent performance far beyond their traditional counterparts [2]. The abundant new physics and novel applications make the "catenary optics" as an important advancement in the field of optics as well as nano-photonics.

Besides the "catenary" shapes, "gammadion" shape has also been used for the design of metasurface. As we know, gammadion is the symbol of Buddhist Associations, which symbolizes the four cardinal corners of this world. Wang et al. [3] reported that gammadion structure embedded with a pair of splits, as a simple and compact structure, can be applied to make efficient optical devices. By purposely changing the relative split positions at certain arms to selective excitation of different resonance modes in the gammadion metamaterials, $\mathrm{THz}$ wave amplitude manipulation, as well as broadband static resonance tunability at the resonance frequency, can be theoretically and experimentally achieved. By combining the concept of catenary structures and gammadion structures, one would be able to design optical devices with complex exotic functionalities. Their methodologies, delivered results, and what's more, the philosophy for getting the inspiration, would stimulate broad interest for the researchers in other field as well.

1 M. B. Pu, X. Li, X. L. Ma, Y. Q. Wang, Z. Y. Zhao, C. T. Wang, C. G. Hu, P. Gao, C. Huang, H. R. Ren, X. P. Li, F. Qin, J. Yang, M. Gu, M. H. Hong, and X. G. Luo, Sci. Adv. 1, e1500396 (2015).

2 X. G. Luo, Sci. China-Phys. Mech. Astron. 58, 594201 (2015).

3 D. C. Wang, Q. Huang, C. W. Qiu, and M. H. Hong, Sci. China-Phys. Mech. Astron. 58, 084201 (2015).

*Corresponding author (email: hbsun@jlu.edu.cn) 